\title{
Prenatal diagnosis of Pallister-Killian syndrome using cord blood samples
}

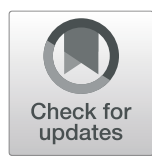

Ting Wang ${ }^{1 *}\left(\mathbb{D}\right.$, Congmian Ren ${ }^{1}$, Dan Chen², Jian Lu', Li Guo ${ }^{1}$, Laiping Zheng ${ }^{1}$, Yuan Liu ${ }^{1}$ and Hanbiao Chen ${ }^{1}$

\begin{abstract}
Background: Pallister-Killian syndrome (PKS) (OMIM:\#601803) is a rare sporadic genetic disorder characterized by multi-malformations which is caused by the presence of the extra isochromosome 12p. PKS is featured by the tissue-limited mosaicism of the isochromosome 12p [i(12p)]. There were a wide spectrum of prenatal ultrasound findings of PKS, which made it difficult to be found in first or second trimester. Polyhydramnios, diaphragmatic hernia, and rhizomelic limb shortening were the most common prenatal ultrasound abnormalities in PKS. This study retrospectively analyzed the ultrasound findings and molecular cytogenetic results of four PKS fetuses diagnosed by using cord blood samples.

Results: The ultrasound anomalies of four PKS fetuses are described as follows: fetal macrosomia, cerebral ventriculomegaly, increased NT thickness, rhizomelic limbs shortening, polyhydramnios. Biparietal diameter (BPD), head circumference $(H C)$, abdominal circumference $(A C)$, femur length (FL) measurements were above the mean in three fetuses, while one fetus showed rhizomelic limbs shortening. Combined with this study and previous literature, polyhydramnios was the most frequent anomaly observed in prenatal ultrasound examination of PKS, which accounted for 48\% (94/194). Fetal macrosomia was present in 15\% (29/194), cerebral ventriculomegaly in 13\% (25/194), thickened nuchal fold in 9\% (18/194), rhizomelic limbs shortening in 26\% (51/194). I(12p) was found in the karyotype analysis of cultured cord blood lymphocytes and the mosaic ratios ranged from 2 to $5 \%$. Single nucleotide polymorphisms array (SNP-array) results suggested that the whole short arm of chromosome 12 was duplicated with 2 3 copies. Fluorescence in situ hybridization (FISH) was performed to confirm the results of karyotype and SNP-array.

Conclusions: In case non-specific indicators such as fetal macrosomia, polyhydramnios and rhizomelic limbs shortening are observed meanwhile in prenatal ultrasound, targeted detection of PKS should be considered. In the prenatal diagnosis of PKS, the combination of SNP-array and FISH with conventional karyotype are the key to seek i(12p) and for precise diagnosis.
\end{abstract}

Keywords: Pallister-Killian syndrome, Prenatal diagnosis, Isochromosome 12p, Cord blood, Ultrasound findings

\section{Background}

Pallister-Killian syndrome (PKS) (OMIM:\#601803) is a rare sporadic genetic disorder characterized by developmental delays, prominent foreheads, sparse frontotemporal hairs, craniofacial deformities, mental retardation, epilepsy, low tension, skin pigmentation, congenital heart defects and other systemic abnormalities. The feature of this syndrome is the presence of isochromosome $12 \mathrm{p}[\mathrm{i}(12 \mathrm{p})]$ mosaicism with tissue-limited. Tissue-limited mosaicism

\footnotetext{
*Correspondence: kevinwtwt@163.com

${ }^{1}$ Medical Genetic Center, Guangdong Women and Children Hospital, 521

Xingnan Avenue, Panyu, Guangzhou, China

Full list of author information is available at the end of the article
}

refers to the differences in chromosome between different tissues in the same individual. The percentage of cells with $\mathrm{i}(12 \mathrm{p})$ depends on the tissue examined, regardless of the severity of the syndrome [1]. The mosaicism rates of skin fibroblasts, amniotic cells or chorionic villus cells are higher than that of rapidly dividing lymphocytes [2]. Over time, tetraploid cells are rapidly replaced by euploidy cells in the peripheral blood, and postnatal infants were usually diagnosed by buccal smear or skin fibroblasts.

Prenatal diagnosis of PKS remains a challenge because of the difficulty in detecting additional $\mathrm{i}(12 \mathrm{p})$ and the diversity of mosaicism level. Moreover, the supernumerary marker chromosomes are easily decreased in amniotic fluid cell

(c) The Author(s). 2019 Open Access This article is distributed under the terms of the Creative Commons Attribution 4.0 International License (http://creativecommons.org/licenses/by/4.0/), which permits unrestricted use, distribution, and 


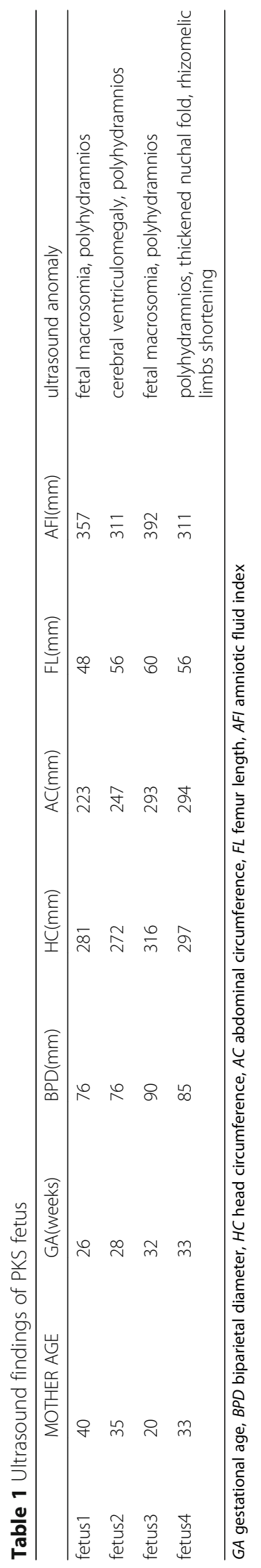




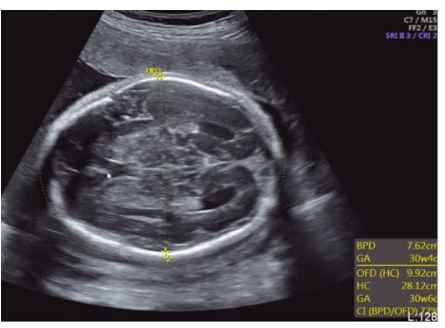

a $\mathrm{BPD} H \mathrm{HC}$ are larger than the gestational age

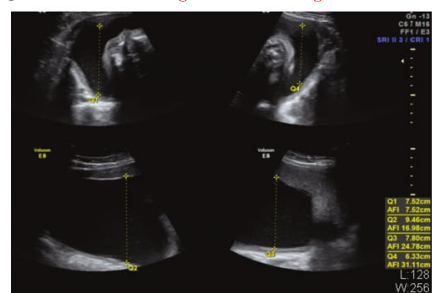

b polyhydramnios

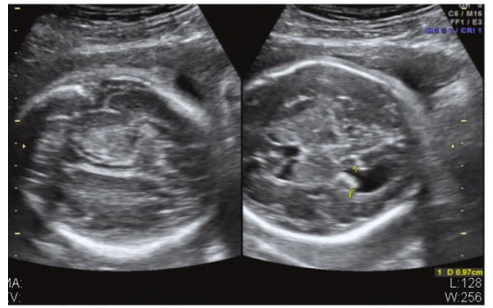

C Left ventricle of fetus approximate to dilatation

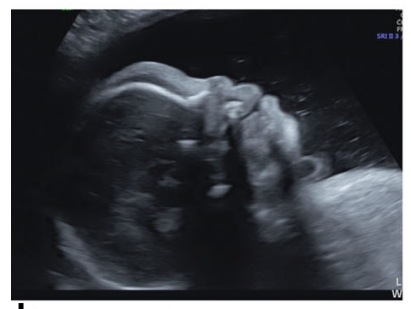

d hydropic forehead

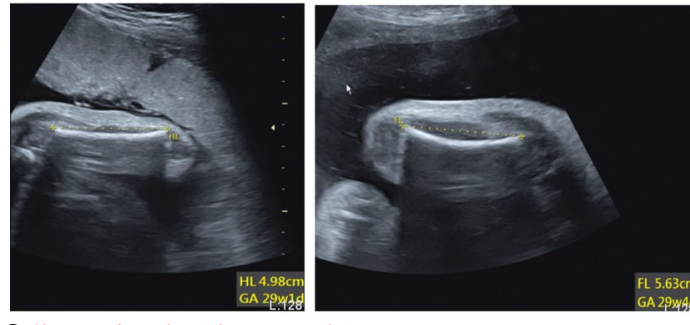

e Shorten length of humerus and femur

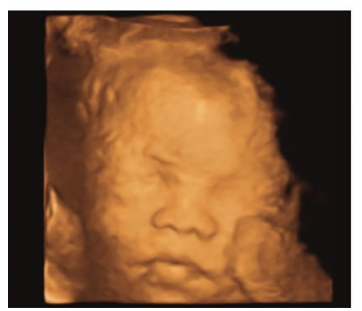

f $3 \mathrm{D}$ image
Fig. 1 Ultrasound image of 4 fetuses with PKS. a: 2 of 4 fetuses' biparietal diameter and head circumference were large for gestational age and (b) polyhydramnios; c: Besides mild polyhydramnios, fetus2's left ventricle approximate to dilatation. d: Fetus4 has hydropic forehead and (e) shorten length of humerus and femur. f: There are no sinificant positive characteristics in all the fetuses'profiles of 3D ultrasound images

culture [3]. Using traditional cytogenetic banding techniques to identify marker chromosomes may be difficult. The most commonly considered prenatal diagnosis usually occurred in cases of congenital diaphragmatic hernia $(\mathrm{CDH})$ or incidental chorionic villus sampling (CVS) or amniocentesis due to advanced maternal age, nonspecific ultrasound abnormalities or even less in the case of prenatal screening abnormalities [4]. There were a wide spectrum of prenatal ultrasound findings in PKS, which made it difficult to be found in first or second trimester. Polyhydramnios, $\mathrm{CDH}$, and rhizomelic limb shortening were the most common prenatal ultrasound abnormalities in PKS [5].

Single nucleotide polymorphisms array (SNP-array) can detect copy number variations using DNA isolated from uncultured specimens [6]. In this study, we presented 4 cases of PKS diagnosed by karyotype and SNParray in second trimester. All the 4 cases were analysed in terms of ultrasound manifestations carefully and compared with the previous literature. To our knowledge, this series is the first report of prenatal diagnosis of PKS in China from a single center.

\section{Methods}

We retrospectively analyzed the ultrasound and genetic results of all fetuses diagnosed with PKS in our medical genetic center between January 2017 and December 2018. The follow-up were performed by experienced nurse. We reviewed the ultrasound findings of PKS published in the last decade in order to identify the anomalies and markers existing in PKS. This study obtained the informed consent of all involved women and was approved by the Ethics Committee of Guangdong Women and Children hospital.

Table. 2 Z-scores and centile of 4 fetuses with PKS

\begin{tabular}{|c|c|c|c|c|c|c|c|c|c|}
\hline & \multirow[b]{2}{*}{ GA } & \multicolumn{4}{|c|}{ Z-scores } & \multicolumn{4}{|c|}{ centile } \\
\hline & & $\mathrm{HC}$ & BPD & $A C$ & $\mathrm{FL}$ & $\mathrm{HC}$ & BPD & $A C$ & $\mathrm{FL}$ \\
\hline fetus1 & 26 & 4.6 & 3 & 1 & 0.6 & 100 & 100 & 85 & 73 \\
\hline fetus2 & 28 & 1.3 & 0.9 & 1.2 & 2.2 & 90 & 80 & 88 & 99 \\
\hline fetus3 & 32 & 2.2 & 2 & 1.4 & 0.2 & 99 & 97 & 91 & 59 \\
\hline fetus4 & 33 & -0.5 & -0.3 & 0.7 & -2.1 & 32 & 39 & 76 & 2 \\
\hline
\end{tabular}

GA gestational age, $B P D$ biparietal diameter, $H C$ head circumference, $A C$ abdominal circumference, $F L$ femur length 


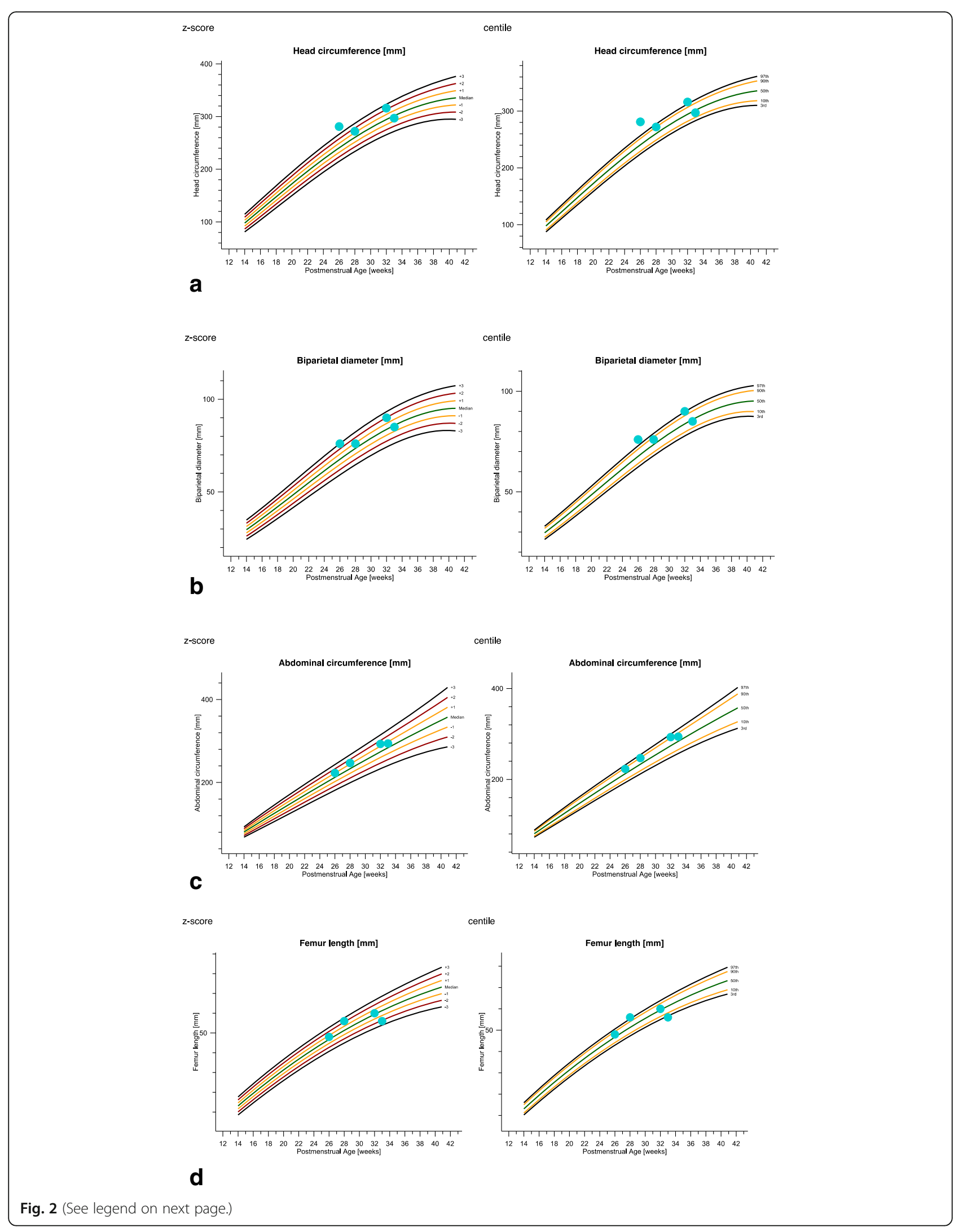


(See figure on previous page.)

Fig. 2 Fetal biometry charts of fetal growth based on INTERGROWTH-21st International Standards for Fetal Growth (v1.6.4). Biparietal diameter,

head circumference, abdominal circumference and femur length measurements were above the mean in three fetuses, while fetus 4 showed

rhizomelic limbs shortening. a: Z-scores and centile of head circumference. b: Z-scores and centile of biparietal diameter. c: Z-scores and centile

of abdominal circumference. $\mathbf{d}$ : Z-scores and centile of femur length

Voluson E8 (GE Healthcare, USA) was applied for ultrasound examinations, which were performed by specialists experienced in fetal medicine. The gestational weeks of all the involved women exceeded 26 weeks when they were referred to our center. Subsequent cordocentesis were performed and $2 \mathrm{~mL}$ cord blood were aspirated into asepsis pipe and sent to the laboratory as soon as possible. All specimens were divided into two parts, one for cell culture and one for molecular genetic testing after DNA isolation using QIAamp DNA Blood Mini Kit (QIAGEN, Germany). NANODROP 2000 (Thermo, USA) was applied to determine DNA concentration. CytoScan $750 \mathrm{~K}$ chip (Affymatrix, USA) was used for SNP-array. All the procedures were operated according to the manufacturer's protocol. The results were analyzed with ChAS3.1.0.15 (Affymatrix, USA) software. The remaining samples were employed for routine cell culturing according to standard culture process. Karyotypes were obtained after GTGbanding. Fluorescence in situ hybridization (FISH) was performed to confirm the diagnosis of PKS using chromosome 12p telomere probe (Abbott Vysis TelVysion 12p Spectrum Green probe, USA) and control chromosome 16 centromere probe (Abbott Vysis CEP 16 Spectrum Orange probe, USA).

\section{Results}

The mean age of the women was 32 years and the mean gestational age was 30 weeks. All the pregnancies were singleton and the women had unremarkable history. Two women were primigravida and the other had healthy children. Anomalies of ultrasound findings were as followed: fetal macrosomia, cerebral ventriculomegaly, increased NT thickness, rhizomelic limbs shortening, polyhydramnios. The descriptions of ultrasound findings were summarized and demonstrated in Table 1 and Fig. 1 . In this study, the most common ultrasound finding was polyhydramnios that affected all 4 fetuses which was consistent with previous literature [3]. Table 2 and Fig. 2 documented the Z-scores and Centile of 4 fetuses with PKS according to International Standards for Fetal Growth (v1.6.4). Biparietal diameter (BPD), head circumference (HC), abdominal circumference (AC) and femur length (FL) measurements were above the mean in three fetuses, while one fetus showed rhizomelic limbs shortening. Combined with our dataset and previous literature [3], polyhydramnios was the most frequent anomaly observed in ultrasound examination which accounted for $48 \%$ (94/194). Fetal macrosomia was present in $15 \%(29 / 194)$, cerebral ventriculomegaly in $13 \%$ (25/
194), thickened nuchal fold in 9\% (18/194), rhizomelic limbs shortening in 26\% (51/194).

Since the gestational weeks were all over 24 weeks, all 4 cases received invasive prenatal diagnosis with cordocentesis. Karyotype, SNP-array and FISH results of 4 fetuses were shown in Fig. 3 and summarized in Table 3 according to the International System for Human Cytogenomic Nomenclature 2016 version (ISCN 2016). Karyotype analysis of cultured cord blood lymphocytes was performed in all cases, and the cells counting was increased to 100 cells as a diagnostic test. De novo supernumerary i(12p) was found in the cultured cord blood lymphocytes and the mosaic ratios ranged from 2 to 5\%. DNA extracted from uncultured cord blood lymphocytes was used for SNP-array detection and the results suggested that the whole short arm of chromosome 12 was duplicated with a copy number between 2 and 3. Interphase FISH was performed to confirm the results of karyotype and SNP-array. The mosaic ratios of $\mathrm{i}(12 \mathrm{p})$ in uncultured cord blood interphase FISH ranged from 22 to $46 \%$. After genetic counseling, all women decided to terminate their pregnancies.

\section{Discussion}

PKS is difficult to detect in prenatal diagnosis because most cases were sporadic. Less than 100 prenatal cases have been published since first described by Gilgenkrantz in a prenatal diagnosis of tetrasomy $12 p$ from amniocytes [7]. Santamaria et al. published a prenatal case report of a fetus with massive diaphragmatic hernia and summarized the main ultrasound indicators of PKS including polyhydramnios, rhizomelic limbs shortening and $\mathrm{CDH}$ [8]. Kucińska-Chahwan et al. believed that the presence of $\mathrm{CDH}$ is sufficient to modify routine to targeted testing for PKS [9]. Mourali et al. presented the first case of early prenatal diagnosis of PKS due to the presence of increased nuchal translucency and $\mathrm{CDH}$ [10]. However, none of the 4 cases we presented had $\mathrm{CDH}$. This deviation likely reflects the diversity of clinical expression of PKS. Doray et al. stated that the most frequent indicator of PKS was polyhydramnios (84\%) [11]. Polyhydramnios seen along with $\mathrm{CDH}$ may be the result of esophageal compression due to hernia [12]. On the contrary, all the fetuses we presented had polyhydramnios but not accompanied with $\mathrm{CDH}$. This indicated that polyhydramnios in PKS may be caused by multi-malformation.

Fetuses with PKS exhibited a very typical growth pattern characterized by increased BPD and HC, usually above the 90th percentile, accompanied with femoral growth delay, 


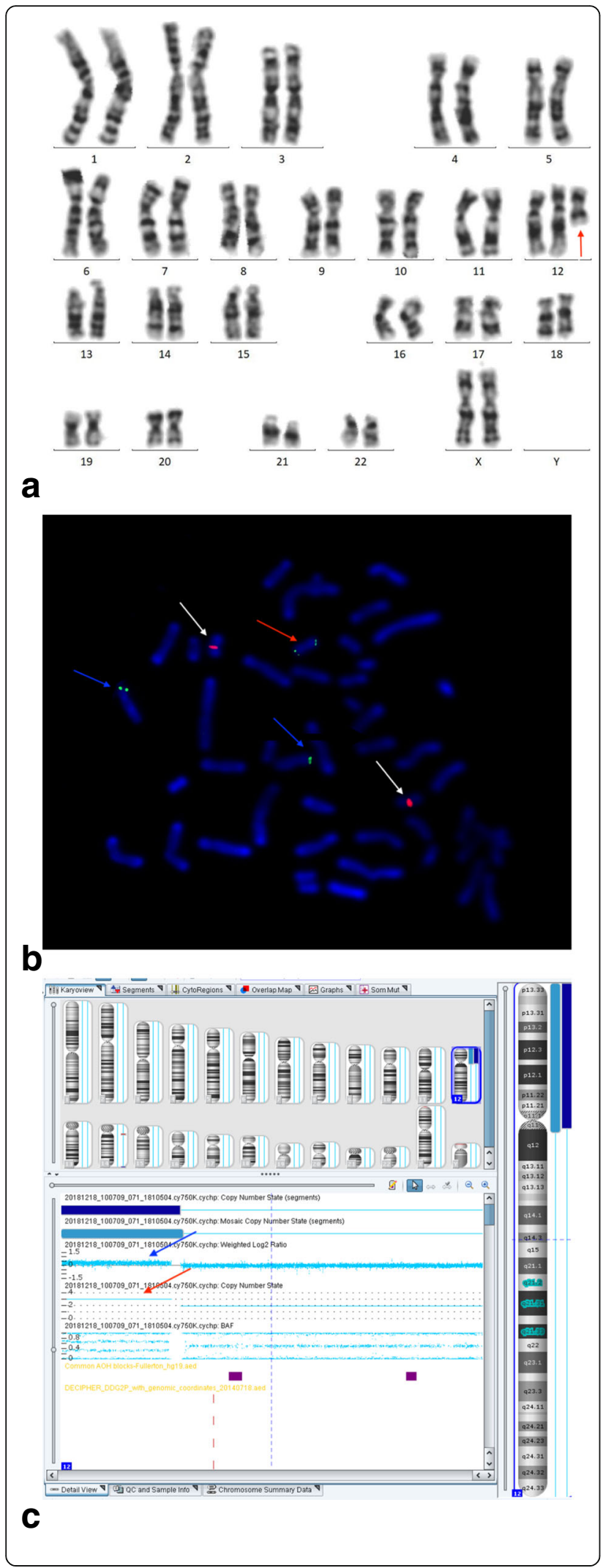

Fig. 3 a: GTG-banding karyotype of PKS fetus in cord blood lymphocytes. Red arrow shows a supernumerary isochromosome 12p. b: Metaphase FISH using 12p telomere probe labeled green and 16 centromere probe labeled orange. Blue arrows show the normal chromosome 12. White arrows show the chromosome 16. Red arrow shows the isochromosome 12p. c: SNP-array analysis of uncultured cord blood. Blue arrow shows the log2 ratio. Red arrow shows the lines of copy number variants of $12 p$ were between 2 and 3

which is significantly below the 10th percentile for gestational age. In our study, the $\mathrm{HC}$ percentile of four fetuses was 100th, 90th, 99th, and 32th respectively. But the percentile of FL was higher than 10th, except that of fetus 4 (2th). This further illustrated the varity of ultrasound manifestations of PKS. Karaman et al. presented 15 children with PKS, two of whom were macrosomia [13]. Salzano et al. summarized all the previously published reports of PKS and obtained the percentage of fetal macrosomia was $14 \%(26 / 190)$ [3]. The ultrasound findings of fetus 1 and fetus 3 were decribed as fetal macrosomia shown in Table 2 due to the high percentile of $\mathrm{HC}$ and $\mathrm{AC}$. Compared with previous literatures, 2 cases of macrosomia were found in 4 cases of PKS fetus in this study, with higher proportion. Although the number of cases was relatively small, it also showed the difficulty of PKS in prenatal diagnosis. In case of the wide phenotypic spectrum of PKS, we strongly recommend that even if each of these abnormal ultrasound findings is non-specific, PKS should be highly suspected when rhizomelic limbs shortening, polyhydramnios and fetal macrosomia are observed simultaneously. Thicken nuchal fold and cerebral ventriculomegaly were also found in our study, which were consistent with the ultrasound findings reported in previous literature $[14,15]$.

PKS is caused by the presence of additional $\mathrm{i}(12 \mathrm{p})$. It is difficult to detect $\mathrm{i}(12 \mathrm{p})$ cells in cord blood due to the low response of lymphocyte in phytohemagglutinin (PHA) stimulation $[16,17]$. I(12p) were usually absence in lymphocytes culture, but could be found in skin fibroblasts and other tissues such as buccal smears, chorionic villi and amniotic cells [18-20]. Four pregnant women had missed the time of amniocentesis when they were refered to our center, so they could only take cordocentesis for prenatal diagnosis. Theisen et al. used genomic DNA to detect $\mathrm{i}(12 \mathrm{p})$ that could not be detected in karyotype analysis of PHA-stimulated blood culture by aCGH [21]. Unlike to previous published literatures, we also performed cytogenetic and molecular analysis of all the four fetuses with PKS using cord blood samples. Since SNP-array results indicated the presence of $12 p$ with 2 to 3 copies, the cell counts of karyotype were expanded to 100 and the tetrasomy cells of $12 p$ were finally found as Fig. 3 shown. FISH on uncultured cord 
Table. 3 Molecular and cytogenetic results of 4 fetuses with PKS

\begin{tabular}{|c|c|c|c|}
\hline & karyotype & SNP-array & FISH \\
\hline tus1 & $\operatorname{mos} 47, X Y,+i(12)(p 10)[5] / 46, X Y[95]$ & 3] 12p13.33p11.1(173786_34835641 & $/ 12 \mathrm{pl}$ \\
\hline fetus2 & $\operatorname{mos} 47, X X,+i(12)(p 10)[3] / 46, X X[97]$ & arr [GRCh38] 12p13. 33p11.1 (173786_34835641) ×2-3 & nuc ish 12p13 $($ TEL $\times 4) / 12 p 13($ TEL $\times 2)[18 / 8$ \\
\hline tus3 & $\operatorname{mos} 47, X X,+i(12)(p 10)[5] / 46, X X[95]$ & arr [GRCh38] 12p13.33p11.1 (173786_34835641) ×2-3 & nuc ish 12p13 $($ TEL $\times 4) / 12 p 13($ TEL $\times 2)[35 / 6$ \\
\hline tus4 & $\operatorname{mos} 47, \mathrm{XX},+\mathrm{i}(12)(\mathrm{p} 10)[2] / 46, \mathrm{XX}[98]$ & $1.1\left(173786 \_34835641\right) \times 2-3$ & nuc ish 12p13 (TEL \\
\hline
\end{tabular}

blood cells confirmed the presence of $12 \mathrm{p}$ mosaicism in four fetuses.The results of SNP-array in cord blood of 4 fetuses showed 2 to 3 copies of $12 p$, which may be due to the neutralization of double dose of $12 p$ in normal cells and quadruple dose of $12 p$ in abnormal cells. In the prenatal diagnosis of PKS, the combination of SNP-array and FISH with conventional karyotype are the key to seek $\mathrm{i}(12 \mathrm{p})$ and for precise diagnosis.

The mechanism of $i(12 p)$ formation is still not fully revealed. Previous literatures have illustrated that maternal meiotic II nondisjunction is a mechanism of tetrasomy 12 mosaicism, although there were several reports of paternal nondisjunction [22, 23]. Similar to other autosomal aneuploidy syndrome, advanced maternal age is a high risk factor. Theoretically, in case a parent had $\mathrm{i}(12 \mathrm{p})$ germline mosaicism or balanced rearrangement on chromosome $12 \mathrm{p}$, there were high risk of recurrence of pregnancy with PKS [24]. The four couples also had no chromosome rearrangements such as cryptical pericentric inversions which could lead to duplication or isochromosome formation of $12 p$ [25].

In conclusion, our study presented the atypical expression of PKS in prenatal diagnosis such as ultrasound finding and diagnostic samples. In case non-specific indicators such as fetal macrosomia, polyhydramnios and rhizomelic limbs shortening are observed meanwhile in ultrasound examination, targeted detection of Pallister-Killian syndrome should be considered, including expanded cell counts in karyotype and using uncultured genomic DNA for molecular detection.

\section{Abbreviations \\ AC: Abdominal circumference; AFI: Amniotic fluid index; BPD: Biparietal diameter; $\mathrm{CDH}$ : Congenital diaphragmatic hernia; FISH: Fluorescence in situ hybridization; FL: Femur length; GTG-banding: Giemsa trypsin banding; HC: Head circumference; i(12p): Isochromosome 12p; \\ PHA: Phytohemagglutinin; PKS: Pallister-Killian syndrome; SNP-array: Single nucleotide polymorphisms array}

\section{Acknowledgements}

We would like to express our sincere gratitude to our patients and their family for their cooperation.

\section{Authors'contributions}

All authors have materially participated in the study and manuscript preparation.

TW analyzed the clinic data, drafted the manuscript; LG and HC carried out the clinic data analysis, and participated in the design of the work; $J$ participated in SNP-array analysis. LZ and YL participated in the karyotyping analysis. CR participated in the FISH.DC participated in the ultrasound findings. All authors have approved the final article.
Funding

Not applicable.

\section{Availability of data and materials}

The datasets used and/or analyzed during the current study are available from the corresponding author on reasonable request.

\section{Ethics approval and consent to participate}

This study was performed with the approval of Medical Ethics Committee of Guangdong Women and Children Hospital (Study\#201901115).

\section{Consent for publication}

All patients in this report provided their consent for publication.

\section{Competing interests}

The authors declare that they have no competing interests.

\section{Author details}

${ }^{1}$ Medical Genetic Center, Guangdong Women and Children Hospital, 521 Xingnan Avenue, Panyu, Guangzhou, China. ${ }^{2}$ Ultrasound Diagnosis Department, Guangdong Women and Children Hospital, 521 Xingnan Avenue, Panyu, Guangzhou, China.

Received: 26 June 2019 Accepted: 30 July 2019

Published online: 30 August 2019

\section{References}

1. Wilkens A, Liu H, Park K, et al. Novel clinical manifestations in Pallister-Killian syndrome: comprehensive evaluation of 59 affected individuals and review of previously reported cases. Am J Med Genet A. 2012;158A:3002-17.

2. Takakuwa K, Hataya I, Arakawa M, et al. A case of mosaic tetrasomy $12 p$ (Pallister-Killian syndrome) diagnosed prenatally: comparison of chromosome analyses of various cells obtained from the patient. Am J Perinatol. 1997;14:641-3.

3. Schubert R, Viersbach R, Eggermann T, et al. Report of two new cases of Pallister-Killian syndrome confirmed by FISH: tissue-specific mosaicism and loss of i(12p) by in vitro selection. Am J Med Genet A. 1997:72:106-10.

4. Salzano E, Raible S, Kaur M, et al. Prenatal profile of Pallister-Killian syndrome: retrospective analysis of 114 pregnancies, literature review and approach to prenatal diagnosis. Am J Med Genet A. 2018;176(12):2575-86.

5. Frisova V, Svobodova IT, Tozzi M, et al. Prenatal diagnosis of Pallister-Killian syndrome in pregnancy with normal CVS result and abnormal ultrasound findings in the second trimester. Taiwan J Obstet Gynecol. 2018;57(5):726-9.

6. Kim KK, Won HH, Cho SS, et al. Comparison of identical single nucleotide polymorphisms genotyped by the GeneChip targeted genotyping $25 \mathrm{~K}$, Affymetrix 500K and Illumina 550K platforms. Genomics. 2009;94(2):0-93.

7. Gilgenkrantz S, Droulle P, Schweitzer M, et al. Mosaic tetrasomy 12p. Clin Genet. 1985;28:495-502.

8. Santamaria A, Laganà AS, Barresi V, et al. Prenatally identified Pallister-Killian syndrome: ultrasound pattern and diagnostic considerations. J Obstet Gynaecol. 2016;36(3):406-7.

9. Kucińska-Chahwan A, Bijok J, Dąbkowska S, et al. Targeted prenatal diagnosis of Pallister-Killian syndrome. Prenat Diagn. 2017;37(5):446-52.

10. Mourali M, El FC, Dimassi K, et al. First trimester diagnosis of PallisterKillian syndrome in a fetus with suggestive abnormalities. Tunis Med. 2010;88(9):666-9.

11. Doray B, Girard-Lemaire F, Gasser B, et al. Pallister-Killian syndrome: difficulties of prenatal diagnosis. Prenat Diagn. 2002;22(6):470-7.

12. Thakur S, Gupta R, Tiwari B, et al. Pallister Killian syndrome: review of fetal phenotype. Clin Genet. 2019;95(1):79-84. 
13. Karaman B, Kayserili H, Ghanbari A, et al. Pallister-Killian syndrome: clinical, cytogenetic and molecular findings in 15 cases. Mol Cytogenet. 2018;1 (1):45

14. Srinivasan A, Wright D. Pallister-Killian syndrome. Am J Case Rep. 2014;15: 194-8.

15. Kolarski M, Joksić $G$, Beres M, et al. Prenatal diagnosis of Pallister-Killian syndrome in young woman: ultrasound indicators and confirmation by FISH. Arch Gynecol Obstet. 2009;279(3):377-9.

16. Izumi K, Krantz ID, et al. Pallister-Killian syndrome. Am J Med Genet Part C Semin Med Genet. 2014;166C:406-13.

17. Reeser SL, Wenger SL. Failure of PHA-stimulated i(12p) lymphocytes to divide in Pallister-Killian syndrome. Am J Med Genet. 1992;42(6):815-9.

18. Libotte F, Bizzoco D, Gabrielli I, et al. Pallister-Killian syndrome: cytogenetics and molecular investigations of mosaic tetrasomy $12 p$ in prenatal chorionic villus and in amniocytes. Strategy of prenatal diagnosis. Taiwan J Obstet Gynecol. 2016:55(6):863-6.

19. Peltomäki P, Knuutila S, Ritvanen A, et al. Pallister-Killian syndrome: cytogenetic and molecular studies. Clin Genet. 1987;31(6):399-405.

20. Mathieu M, Piussan C, Thepot F, et al. Collaborative study of mosaic tetrasomy $12 p$ or Pallister-Killian syndrome (nineteen fetuses or children). Ann Genet. 1997:40:45-54.

21. Theisen A, Rosenfeld JA, Farrell SA, et al. aCGH detects partial tetrasomy of $12 p$ in blood from Pallister-Killian syndrome cases without invasive skin biopsy. Am J Med Genet A. 2010;149A(5):914-8.

22. Shen JD, et al. Pallister-Killian syndrome: meiosis II non-disjunction may be the first step in the formation of isochromosome 12p. Chin Med J. 2010; 123(23):3482-5.

23. de Ravel TJ, et al. Post-zygotic origin of isochromosome 12p. Prenat Diagn 2004;24(12):984-8.

24. Izumi $K$, Zhang Z, Kaur M, et al. 12p microRNA expression in fibroblast cell lines from probands with Pallister-Killian syndrome. Chromosom Res. 2014, 22(4):453-61.

25. Conlin LK, Kaur M, Izumi K, et al. Utility of SNP arrays in detecting, quantifying, and determining meiotic origin of tetrasomy $12 \mathrm{p}$ in blood from individuals with Pallister-Killian syndrome. Am J Med Genet A. 2012; 158A(12):3046-53.

\section{Publisher's Note}

Springer Nature remains neutral with regard to jurisdictional claims in published maps and institutional affiliations.

Ready to submit your research? Choose BMC and benefit from:

- fast, convenient online submission

- thorough peer review by experienced researchers in your field

- rapid publication on acceptance

- support for research data, including large and complex data types

- gold Open Access which fosters wider collaboration and increased citations

- maximum visibility for your research: over $100 \mathrm{M}$ website views per year

At $\mathrm{BMC}$, research is always in progress.

Learn more biomedcentral.com/submissions 